\title{
Pathogenesis of experimental pulmonary alveolar proteinosis
}

\author{
B. COR R I N a d E. KING \\ Department of Morbid Anatomy, St. Thomas's Hospital Medical School, London, S.E.1, and the \\ Occupational Hygiene Service, Manchester University
}

\begin{abstract}
Rats exposed to various airborne dusts developed a condition identical to pulmonary alveolar proteinosis as seen in man. The experimental condition developed through a stage of endogenous lipid pneumonia, characterized by numerous large foamy macrophages widely distributed throughout the lung. These cells broke down to release a finely granular material which finally condensed to reproduce the appearances of alveolar proteinosis. Electron microscopy indicated that the alveolar material was produced by type II pneumocytes and may therefore represent pulmonary surfactant. A study of the dust-handling mechanism showed that in affected animals macrophage mobility was seriously impaired.
\end{abstract}

Recent experiments comparing the effect of various dusts on the rat lung have been complicated by a process which as it advances reproduces the histological appearances of pulmonary alveolar proteinosis (Rosen, Castleman, and Liebow, 1958). This complication was first encountered in rats exposed to an aluminium dust cloud (Corrin, 1962). A preliminary report of its presence in further dust inhalation experiments (Corrin and King, 1966) stimulated its retrospective recognition by Gough (1967), and the condition has since been reported by others (Heppleston, 1967 ; Gross and deTreville, 1968a). This paper considers the development of experimental alveolar proteinosis, the efficiency of the pulmonary clearance mechanism in this condition, and the nature of the alveolar material as revealed by electron microscopy.

\section{MATERIALS AND METHODS}

Alveolar proteinosis has been observed in the final stage of two sets of experiments, both designed to test the fibrogenicity of various airborne dusts in the rat lung. In the first experiment, 10 standard laboratory albino rats were exposed to Pyro aluminium powder for 600 hours over a five-month period and were killed with ether at $6,7,10$, and 13 months. In the second experiment, four groups, each comprising 30 specific pathogen-free Wistar strain rats, were exposed to pure quartz, quartz of $60 \%$ and $5 \%$ cristobalite content, and a non-fibrogenic feldspar, for 3,000 hours over an eight-month period. Four similar groups exposed under identical conditions to various quartz modifications including the same pure quartz did not contract alveolar proteinosis. Two ळे groups of 24 rats each served as controls and $\stackrel{\circ}{2}$ remained free of disease. The animals were killedō with coal gas at monthly intervals up to 27 months. Details of the dusts, dust exposure apparatus, dust concentrations, and chemical methods are given else-․․ where (Corrin, 1962; Corrin and King, 1969; Corrin, Flindt, and King, 1970).

At death the lungs were gently distended in situ with $4 \%$ formaldehyde and the entire thoracic con-o tents were then placed in an excess of fixative, $\underline{3}$. Paraffin sections of a coronal slice of each lung and of the hilar lymph nodes were stained with haema- 3 toxylin and eosin, Masson's trichrome, sudan black for bound lipid, by Gordon and Sweet's method for reticulin and by the periodic acid-Schiff (PAS) $\frac{1}{2}$ method. Frozen sections were stained for neutral lipid with sudan red and by the Schultz reaction for N cholesterol. For electron microscopy small pieces of fresh lung were immersed in cold $\left(4^{\circ} \mathrm{C}\right.$.) $4 \%$ paraformaldehyde in cacodylate buffer $(p H$ 7.4) for one $N$ hour, followed by cold osmium tetroxide in veronalo buffer $(p \mathrm{H} \mathrm{7.4)}$ containing $1 \%$ sucrose for a further hour. The tissues were embedded in maraglas. Ultra- $-\frac{C}{0}$ microtomy proved impossible with glass and a黑 diamond knife was required. The difficulty in sectioning was encountered even in relatively dust-free $-{ }^{-}$ areas of the lung and appeared to be due to the $\overrightarrow{\mathbb{D}}$ alveolar proteinosis material as much as to the dusts. $\frac{?}{\Phi}$ Thin sections were stained with uranium acetate and $\mathscr{\complement}$ lead citrate and examined in a Siemens Elmiskop IO electron microscope. 
RESULTS

In the final stages of the experiments, areas of the lung were observed in which the alveoli were filled with a finely granular eosinophilic deposit (Fig. 1). This contained occasional cholesterol crystals, dust particles and phagocytes with an abundant eosinophilic cytoplasm similar in appearance to the surrounding alveolar material. The alveolar deposit was strongly PAS-positive (diastase-resistant). Apart from the presence of dust the appearances were identical to those described in man by Rosen et al. (1958).

The development of this alveolar proteinosis could be traced from its earliest stages in those experiments in which the animals were killed successively from the beginning of the dust exposures. In all dusted animals there was an increase in alveolar macrophages, but this was more marked in those groups which later developed alveolar proteinosis. Early animals from these groups showed particularly large numbers of macrophages, which had an unusually swollen cytoplasm. These cells maintained a fairly diffuse distribution in the lungs. They progressively increased in number and size, assuming a large vacuolated or foamy appearance (Fig. 2). Wide ill-defined areas were affected throughout the lungs, which to the naked eye showed large chalky grey or yellow patches on the pleural and cut surfaces. The appearances at this stage were those of endogenous lipid pneumonia (Gross, Brown, and Hatch, 1952 ; Corrin and King, 1969) which has also been termed pulmonary lipidosis (Jennings, Gresham, and Howard, 1965) and multifocal histiocytosis (Yang, Yang, and Grice, 1966). Next, the macrophages broke down, releasing a loose faintly eosinophilic floccular material into the alveoli (Fig. 3). The cell margins of the macrophages often appeared indistinct and there was a marked resemblance of the extra-cellular material to the swollen macrophage cytoplasm. Cholesterol crystals were found in the free material, and occasionally cholesterol pneumonitis with focal interstitial fibrosis was observed. More frequently, the cholesterol excited no inflammatory reaction and the alveolar walls remained thin with no evidence of fibrosis or cellular infiltration. Sudan red stains were weakly positive. Sudan black gave a stronger reaction and petroleum ether extraction reduced the dry weight of the lungs by $30 \%$ (controls $0.2 \%$ ). The faintly eosinophilic material was finely granular, weakly positive with PAS and light green with Masson's tri-

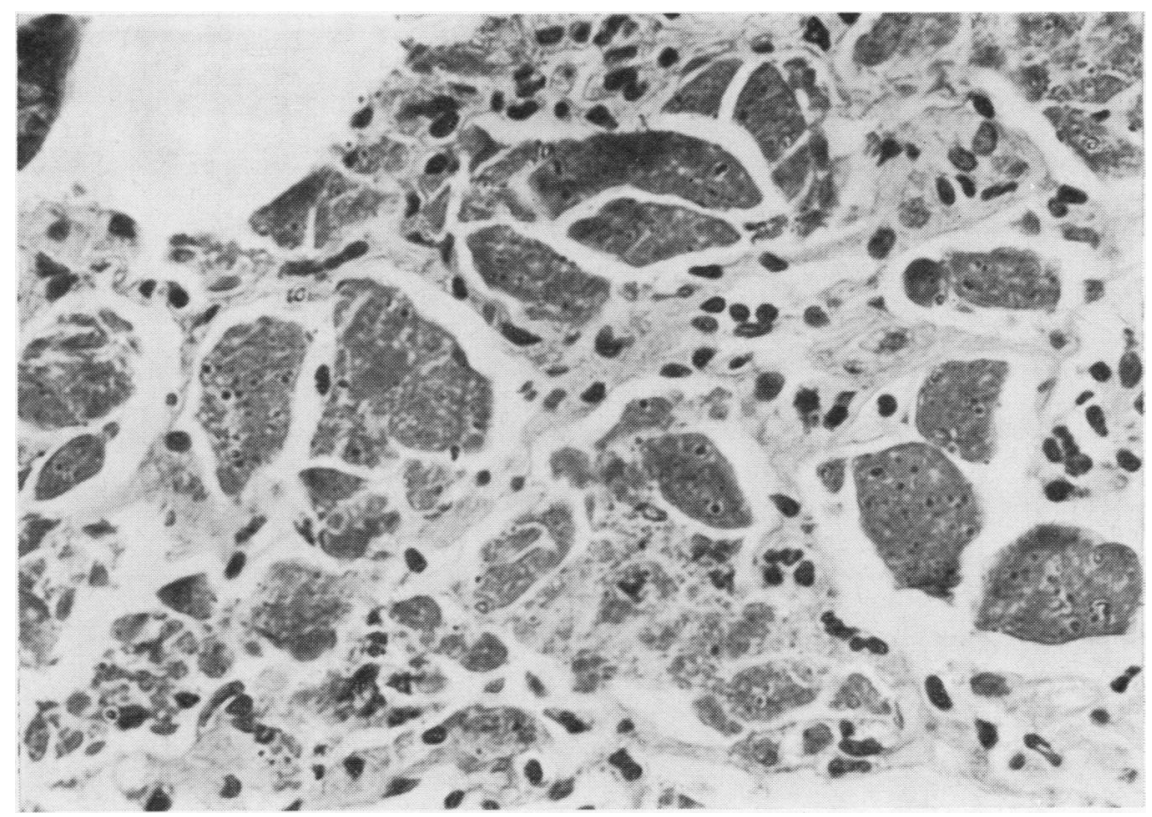

FIG. 1. A strongly PAS positive deposit containing cholesterol crystal clefts fills the aveoli, reproducing the appearances of alveolar proteinosis. Rat lung 23 months after the commencement of feldspar exposure. PAS $\times 480$. 


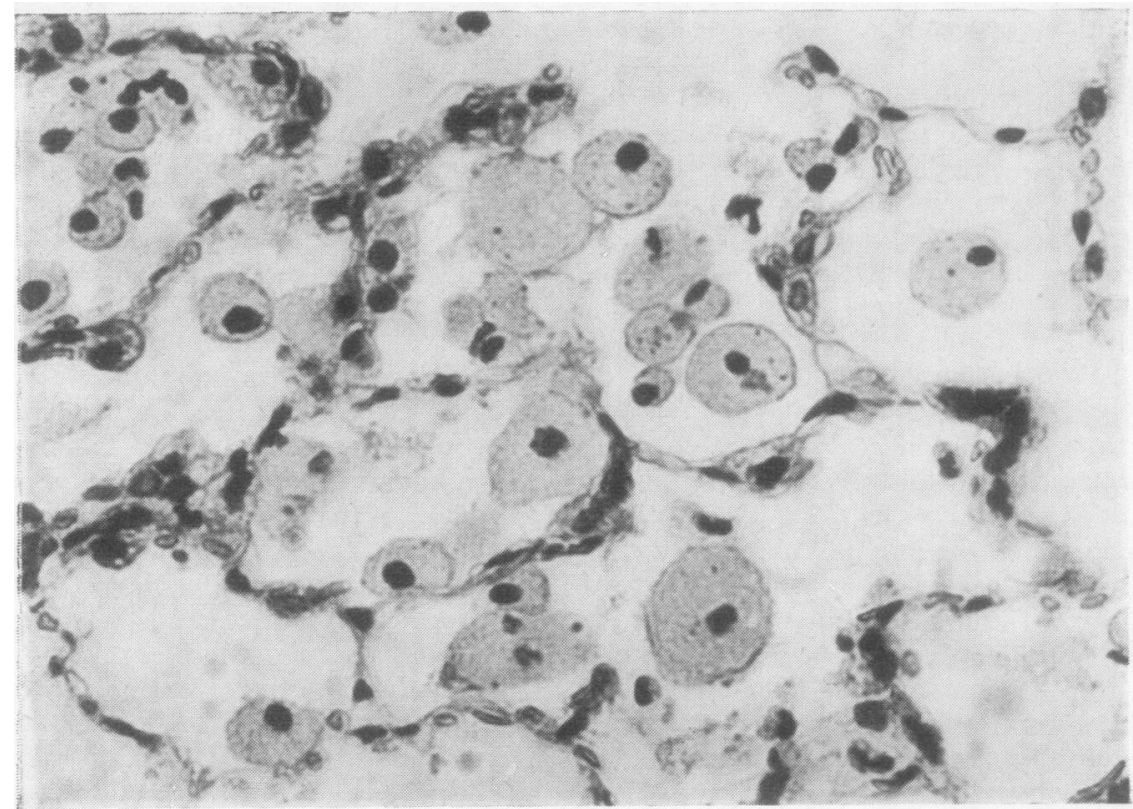

FIG. 2. The alveoli contain many large foamy macrophages. Rat lung after 5 months' exposure to $60 \%$ cristobalite content quartz. $H$. and $E . \times 480$.

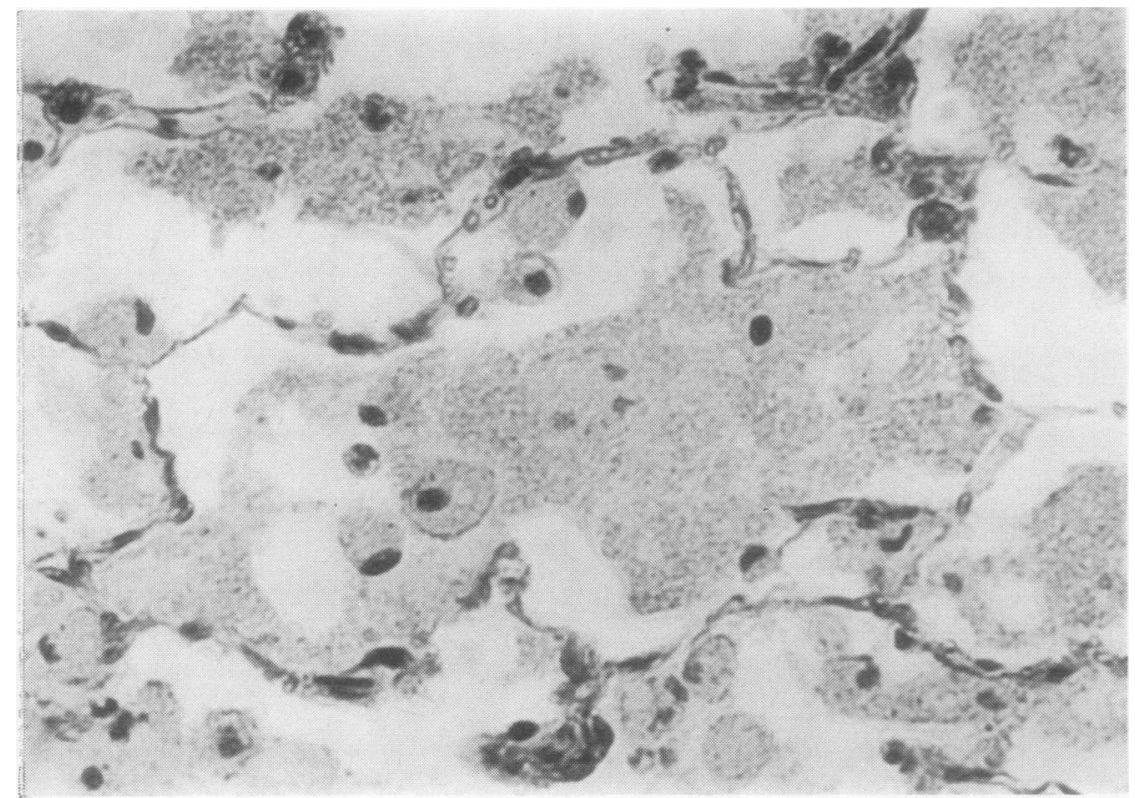

FIG. 3. A loose faintly staining deposit similar in appearance to the cytoplasm of neighbouring foam cells is seen in the alveolar space. Rat lung 23 months after the commencement of cristobalite and quartz exposure. $H$. and $E . \times 480$. 


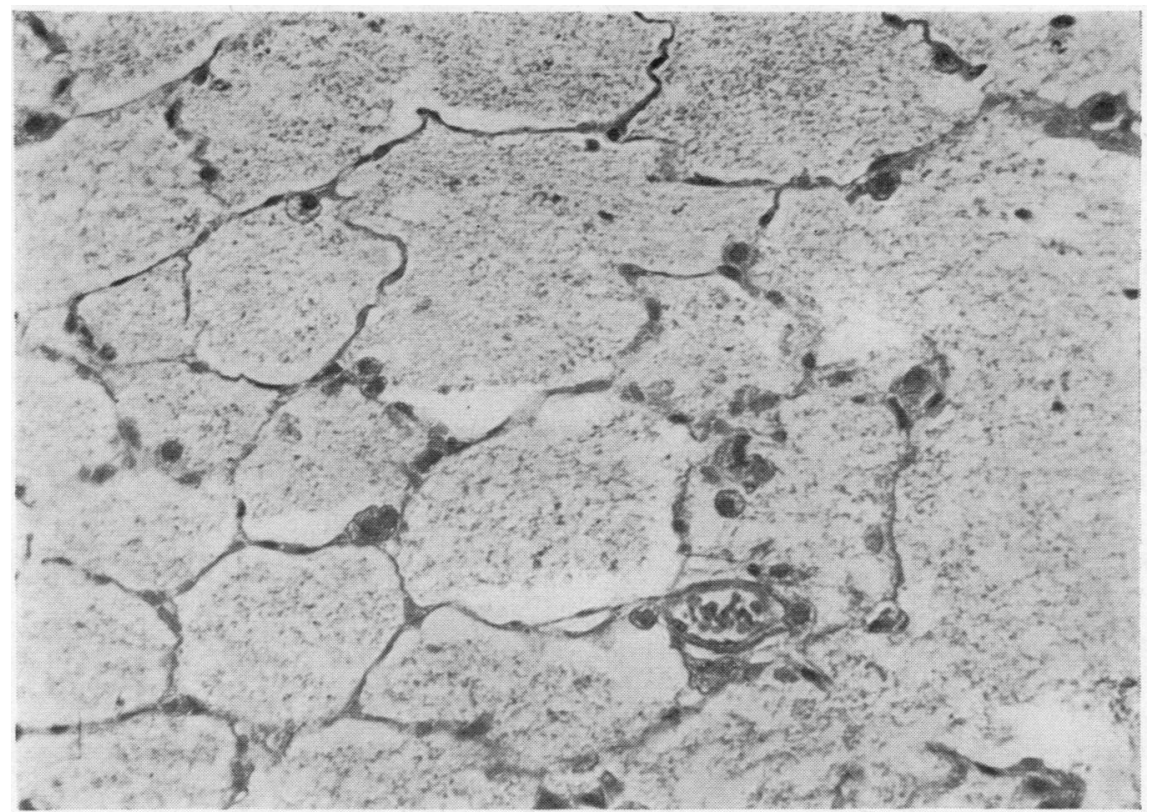

FIG. 4. The finely granular but still loose deposit comes to fill the alveoli. Condensation of this material leads to the appearances seen in Fig. 1, 20 months after the commencement of quartz exposure. $H$. and $E . \times 480$.

chrome stain. It gradually increased in amount (Fig. 4) and became more compact. Concomitant with this it assumed a more deeply eosinophilic appearance, stained bright green with Masson's trichrome and became strongly PAS positive. By this time the appearances of alveolar proteinosis already described were reproduced (Fig. 1).

Whereas affected rats maintained a diffuse distribution of swollen macrophages, in unaffected animals relatively fewer phagocytes were able to concentrate the inhaled dust at the centres of the lung lobules (Macklin's dust sumps). Moreover, these macrophages were unaltered in appearance apart from containing dust and did not have an abundant foamy cytoplasm. As lipid pneumonia and alveolar proteinosis developed it became apparent that these conditions had a retarding effect on the development of silicosis. In unaffected animals a focal concentration of dust proved to be an essential first step in the development of the silicotic nodule. In affected animals this local concentration was delayed or prevented and areas of the lung affected by alveolar proteinosis were noticeably free of silicosis. Silicotic nodules developed in other areas of the lung, and alveolar proteinosis and silicosis generally showed an inverse relationship. The reason for this appeared to be a failure of the foamy macrophages to achieve a high local concentration of silica. Even where the two conditions were closely associated, dust cells in the silicotic nodules differed from neighbouring foam cells in being smaller, non-vacuolated and containing more dust. Transport of dust to the hilar lymph nodes and subsequent fibrosis were facilitated by the presence of lipid pneumonia, but in the lymph nodes the characteristic foam cells seen in the lung were absent. Small compact macrophages were involved in the transport of dust to the lymph nodes and the larger foamy macrophages appeared to be relatively immobile. The contrasting appearances of these cells is shown in Figure 5.

Electron microscopy showed that the finely granular PAS-positive alveolar material was composed of numerous osmiophilic bodies ranging from 0.2 to $2.5 \mu \mathrm{m}$. in diameter, each consisting of many wavy lamellae approximately $5 \mathrm{~nm}$. in thickness (Fig. 6). Sometimes the lamellae had a smoothly curved parallel configuration and occasionally an osmiophilic lattice structure was seen. The free lamellar structures filling the alveoli were identical to the specific cytosomes of the type II 


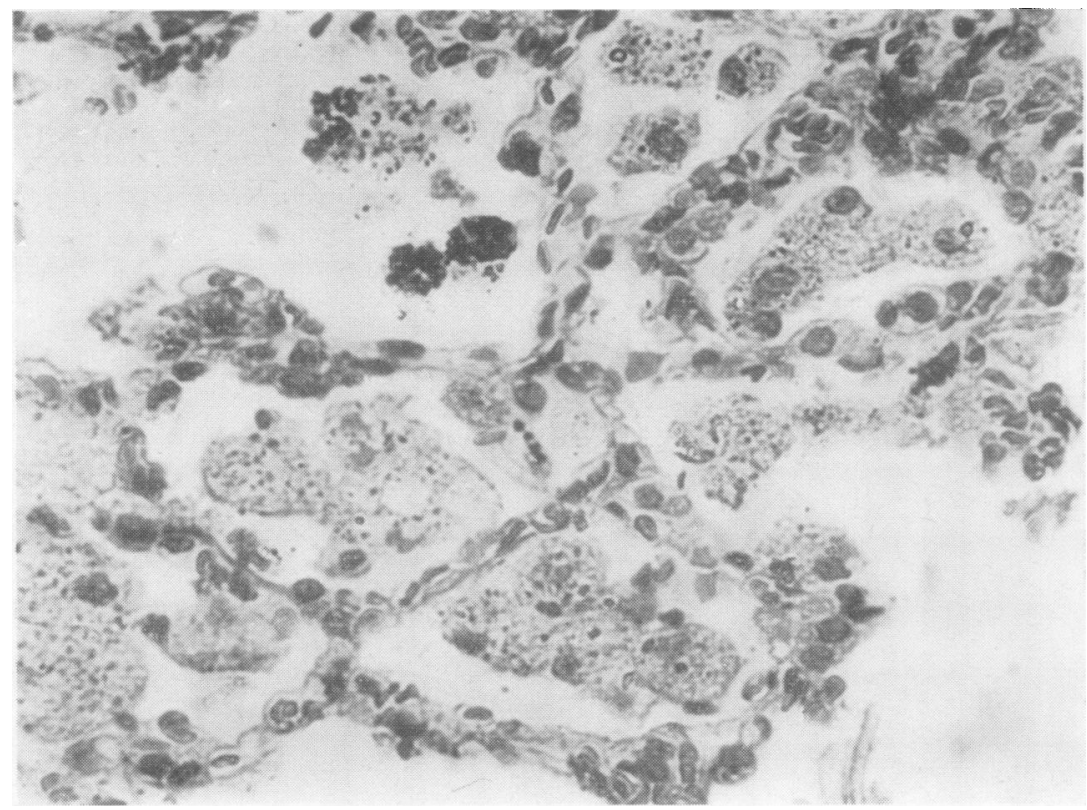

FIG. 5. The alveolus at upper left contains small macrophages heavily charged with dust. Concentration of such cells and their migration to the lymph nodes lead to silicosis. The other alveoli contain larger foamy macrophages which contain less dust and are relatively immobile. They maintain a diffuse distribution in the lung and are not seen in the lymph nodes. $H$. and $E . \times 480$.

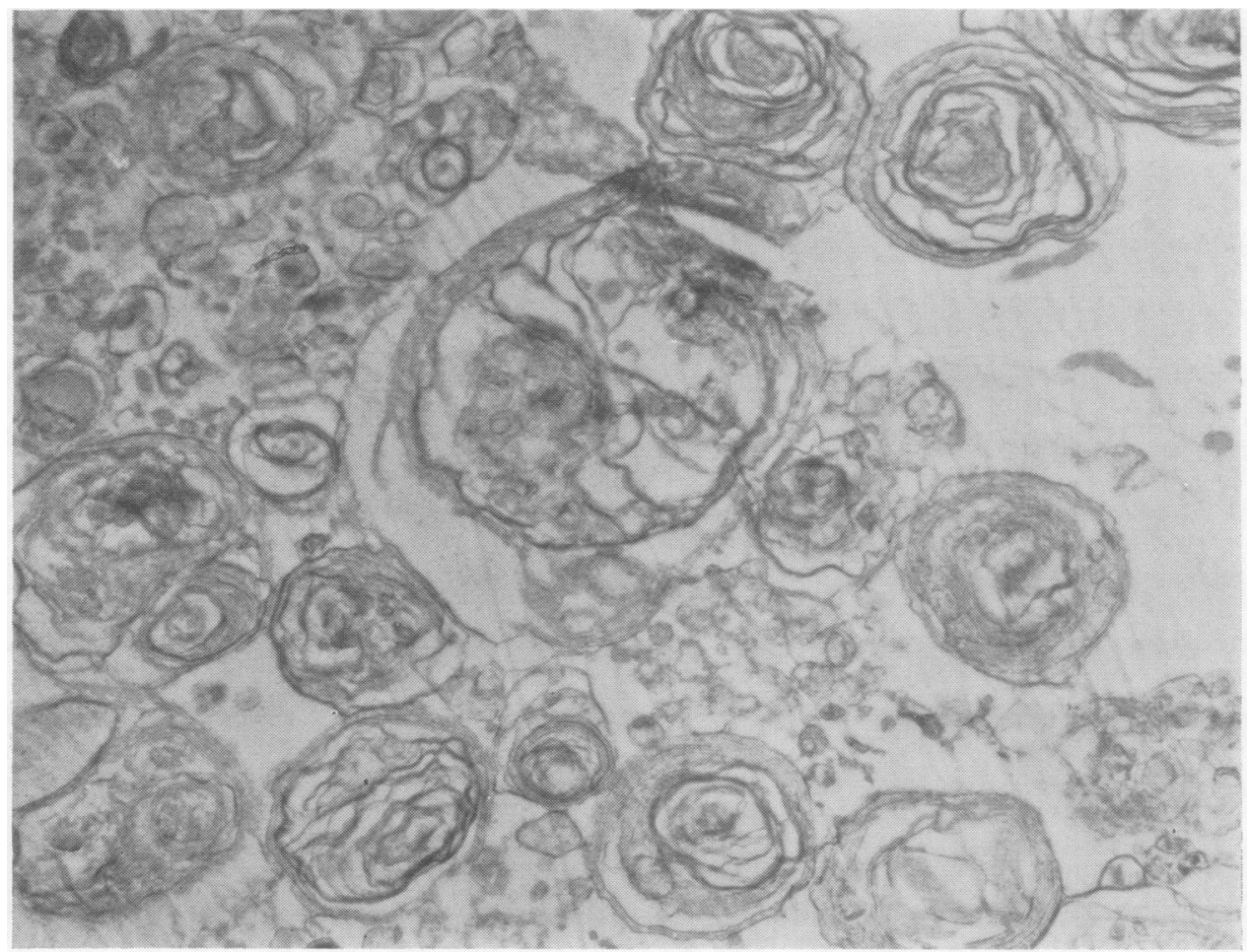

FIG. 6. Electron microscopy shows the finely granular alveolar deposit to consist of numerous osmiophilic lamellar bodies. Uranyl acetate and lead citrate $\times 16,000$. 


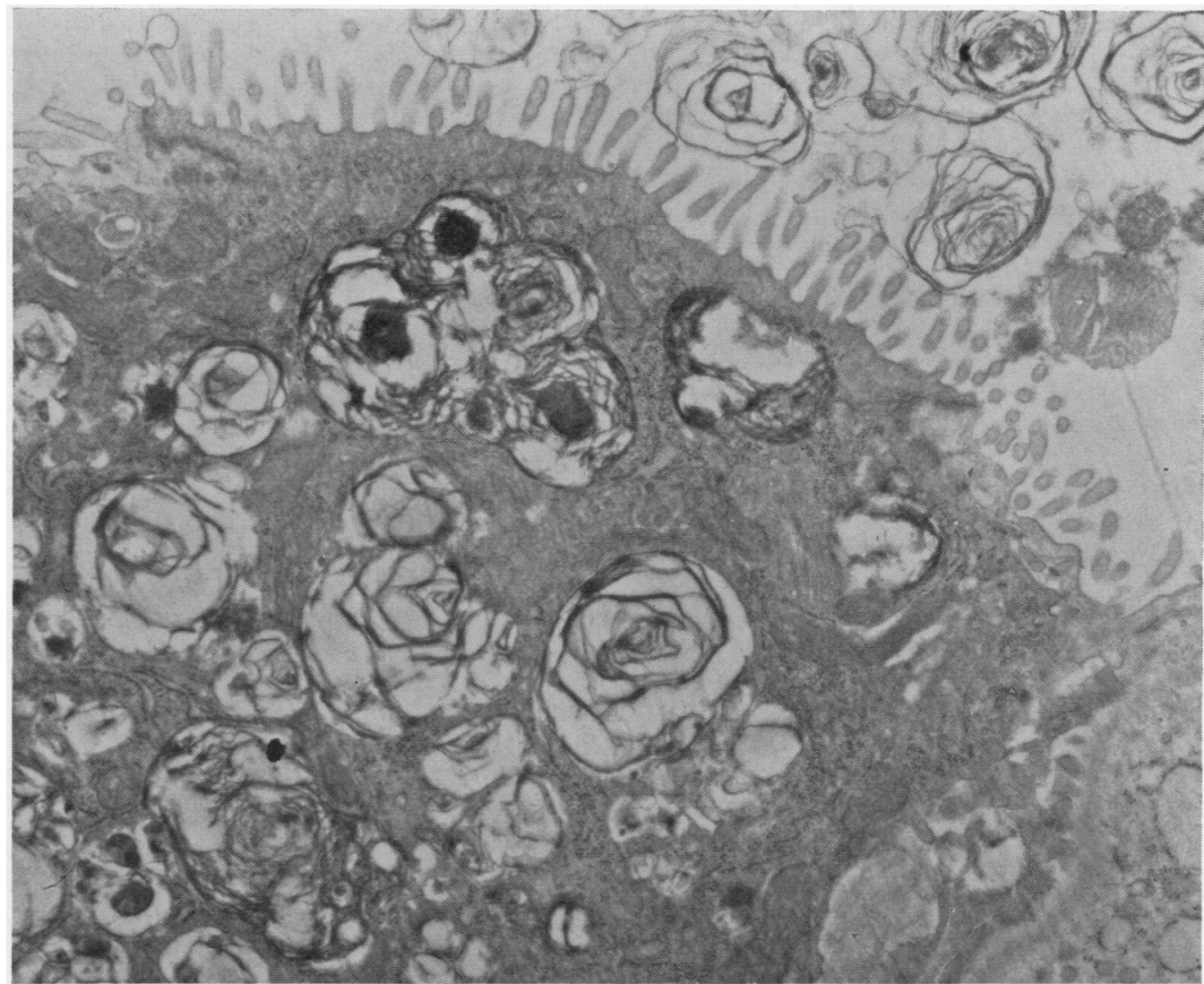

FIG. 7. A type II pneumocyte. Its lamellar inclusions, which are possibly increased in this cell, are believed to represent surfactant secretion. Their similarity to those bodies free in the alveolus is readily apparent. Uranyl acetate and lead citrate $\times 16,000$.

alveolar epithelial cell (Fig. 7). These cells were slightly increased in size, and possessed many lamellar vacuoles up to $2 \mu \mathrm{m}$. in diameter and an abundant rough surfaced endoplasmic reticulum. Their free surfaces bore microvilli and they showed no evidence of degeneration or desquamation. Type I alveolar epithelial cells appeared normal. Free alveolar cells were generally very large and their cytoplasm completely filled with the same wavy lamellar structures seen free in the alveoli and in type II cells. Few other cytoplasmic components could be recognized in the large free cells and it was difficult to identify the cell type. Smaller cells with fewer lamellar bodies were clearly macrophages, however, having long pseudopodia rather than microvilli, other inclusion bodies and little rough endoplasmic reticulum. The source of the lamellar bodies within the macrophages and free in the lumen was clearly the type II pneumocyte. This stimulated a reexamination of the light microscopic sections when occasional areas were found in which type II pneumocytes were unduly prominent, but this appeared to be a relatively late change.

\section{DISCUSSION}

Comparison of the experimental condition with Rosen's (Rosen et al., 1958) original description of alveolar proteinosis leaves little doubt that we are dealing with the same disease. Thus the alveolar deposit is not completely amorphous but finely granular and its lamellar nature, so obviously revealed by electron microscopy, was recognized in the larger granules by Rosen and his colleagues using the light microscope. Strong PAS 
positivity is characteristic of both the experimental and human lesion. In both, lipid stains are positive but, as observed by the original investigators, they give only a poor indication of the large amounts of chemically extractable lipid. Cholesterol crystals constitute part of the lipid. In the early stages, at least, there is little thickening or cellular infiltration of alveolar walls, but interstitial fibrosis may supervene.

Rosen and his colleagues suggested that the characteristic alveolar material was formed by a transformation of large alveolar wall cells, the socalled 'septal cells'. Electron microscopy of the experimental condition supports this origin; to be precise the material is derived from the type II alveolar epithelial cell. These workers further indicated that the 'septal cells' slough off into the alveoli, but our findings do not lend this support. We found no evidence that the type II cells underwent desquamation or were degenerate. The lamellar cytosomes are believed to represent pulmonary surfactant (Klaus, Reiss, Tooley, Piel, and Clements, 1962 ; Buckingham, McNary, and Sommers, 1964) and are normally extruded into the alveolus (Bensch, Schaefer, and Avery, 1964). This process appears to be accentuated in alveolar proteinosis, which may therefore represent an accumulation of pulmonary surfactant. This is supported by a marked increase in palmityl lecithin in the lung washings of patients with alveolar proteinosis (Ramirez-R and Harlan, 1968). In the early stages the extruded lamellar bodies are rapidly taken up by alveolar macrophages and become the predominant ultrastructural component of these cells, which therefore resemble desquamated type II cells on light microscopy. That the free foamy cells are in fact macrophages is indicated by their electron microscopic appearances, the presence of ingested dust and their high content of acid phosphatase (Jennings et al., 1965 ; Gellatly, J. B. M., personal communication, 1968). As the foamy appearance is due to the cells' normal phagocytic properties it is not necessarily evidence of degeneration, but eventually the foamy phagocytes disintegrate, releasing their content of ingested but unaltered lamellar bodies back into the alveoli. Before this they round up, and a study of the silicotic process shows that their mobility is severely diminished.

The accumulation of epithelial cell cytosomes within the alveolus could be due to either hyperactivity of the type II cells or failure of the normal pulmonary clearance mechanism. Rosen and his colleagues noted an increase in alveolar lining cells and the presence of occasional mitoses. We found a similar increase in type II cells as a relatively late feature. Ramirez-R and Harlan (1968) have shown that lipid metabolism is not increased in patients with alveolar proteinosis, and these workers favour an impairment in the removal or degradation process. In our material the presence of dust has facilitated the study of macrophage movement within the lung and towards the hilar lymph nodes. Once the macrophages become distended with the lamellar lipids they round up and their mobility is seriously impaired. They contain dust but are unable to contribute to its usual accumulation at the apex of the lung lobule or its transport to the lymph nodes. This aspect of our findings supports an impairment in the clearance mechanism. Once initiated the lesion would be self-perpetuating by virtue of the impaired macrophage mobility, and in support of this we found that the experimental condition progressed after cessation of dust exposure. The retardation of the silicotic process is explained by this failure to achieve high local concentrations of the inhaled dusts, Gross and deTreville (1968b), however, advance an alternative view which supposes that the dust particles are protected by a lipid coating.

\section{REFERENCES}

Bensch, K., Schaefer, K., and Avery, M. E. (1964). Granular pneumocytes: electron microscopic evidence of their exocrinic function. Science, 145, 1318.

Buckingham, S., McNary, W. F., and Sommers, S. C. (1964). Pulmonary alveolar cell inclusions: their development in the rat. Ibid., 145, 1192.

Corrin, B. (1962). Aluminium pneumoconiosis. M.D. thesis, Univer sity of London.

- and King, E. (1966). Inhalation experiments comparing the action of quartz and cristobalite on the rat lung. Proc. 113th meeting Pathological Society, p. 14 (issued with J. Path. Bact. vol. 92). (1969). Experimental endogenous lipid pneumonia and silicosis. J. Path., 97, 325.

Flindt, M. L. H., and King, E. (1970). In preparation.

Gough, J. (1967). Silicosis and alveolar proteinosis. Brit. med. J. 1, 629.

Gross, P., Brown, J. H. U., and Hatch, T. F. (1952). Experimental endogenous lipoid pneumonia. Amer. J. Path., 28, 211 and deTreville, R. T. P. (1968a). Alveolar proteinosis. Its experimental production in rodents. Arch Path., 86, 255. Hlth, 17, 720.

Heppleston, A. G. (1967). Atypical reaction to inhaled silica. Nature $N$ (Lond)., 213, 199.

Jennings, I. W., Gresham, G. A., and Howard, A. N. (1965). Pul- $\omega$ monary lipidosis in laboratory rats. $J$. reticuloendoth. Soc.,

Klaus, M., Reiss, O. K., Tooley, W. H., Piel, C., and Clements J. A. (1962). Alveolar epithelial cell mitochondria as source of the surface-active lung lining. Science, 137, 750.

Ramirez-R, J., and Harlan, W. R. (1968). Pulmonary alveolar proteinosis. Nature and origin of alveolar lipid. Amer. J. Med., protein 502 .

Rosen, S. H., Castleman, B., and Liebow, A. A. (1958). Pulmonary alveolar proteinosis. New Engl. J. Med., 258, 1123.

Yang, Y. H., Yang, C. Y., and Grice, H. C. (1966). Multifocal histiocytosis in the lungs of rats. J. Path. Bact., 92, 559. 